\title{
The Mediating Role of Hope in the Relationship Between Perceived Stress and Post-Traumatic Stress Disorder Among Chinese Patients with Oral Cancer: A Cross-Sectional Study
}

This article was published in the following Dove Press journal:

Cancer Management and Research

\author{
Ying Zhang' \\ Chunying Cui \\ Lie Wang' \\ Xiaosong $\mathrm{Yu}^{2}$ \\ Yu Wang' \\ Xiaoxi Wang $\mathbb{D}^{3}$ \\ 'Department of Social Medicine, School \\ of Public Health, China Medical \\ University, Shenyang, Liaoning, People's \\ Republic of China; ${ }^{2}$ Department of \\ General Practice, The First Affiliated \\ Hospital, China Medical University, \\ Shenyang, Liaoning, People's Republic of \\ China; ${ }^{3}$ Medical Basic Experimental \\ Teaching Center, China Medical \\ University, Shenyang, Liaoning, People's \\ Republic of China
}

\begin{abstract}
Purpose: Cancer diagnosis and treatment are long-term traumatic stressors. Depression and anxiety are known to be prevalent in patients with cancer, but post-traumatic disorder (PTSD) has been overlooked frequently in this population. This study aimed to estimate the prevalence of PTSD and examined the mediating role of hope in the relationship between perceived stress and PTSD symptoms in Chinese patients with oral cancer.

Methods: A total of 230 oral cancer patients were recruited to complete a questionnaire including the Posttraumatic Stress Checklist-Civilian Version (PCL-C), the Perceived Stress Scale-10 (PSS-10) and the Herth Hope Index (HHI). Analysis of variances (ANOVA) $/ t$-test, Person's $r$ and hierarchical linear regression analysis methods were conducted to assess the associations among perceived stress, hope and PTSD symptoms. Asymptotic and resampling strategies were used to explore the mediating role of hope.

Results: The prevalence of PTSD symptoms was $6.05 \%$ in Chinese patients with oral cancer. Perceived stress was positively related to PTSD symptoms, explaining $39.9 \%$ of the variance. In addition, hope was negatively related to PTSD symptoms, explaining 5.9\% of the variance. Besides, the proportion of mediation of hope was $30.06 \%$.

Conclusion: Perceived stress was positively associated with PTSD symptoms, and hope was negatively associated with PTSD symptoms. Furthermore, hope played partial mediating role in the relationship between perceived stress and PTSD symptoms. Thus, more attention should be paid to patients' PTSD status, and take measures is to relieve stress and increase hope. Keywords: oral cancer, hope, perceived stress, post-traumatic disorder, PTSD
\end{abstract}

\section{Introduction}

Oral cancer is a general term for malignant tumours occurring in the oral cavity and oropharynx. ${ }^{1}$ Because of tobacco and alcohol use, squamous cell carcinoma (SCC) has become increasingly common, accounting for more than $90 \%$ of all oral cancers. ${ }^{2}$ Cancer of the oral cavity is one of the most common malignancies, especially in developing countries. ${ }^{2}$ Worldwide, 405,000 new cases of oral cancer are anticipated each year. ${ }^{3}$ In China, there are more than 46,000 new cases of oral cavity cancer (including lip and pharynx) diagnosed every year, and this cancer causes 21,000 deaths each year. ${ }^{4}$

Oral cavity cancer is a highly lethal disease with a mortality rate that approaches $50 \%$ and it has a high risk of recurrence. ${ }^{5}$ In addition to the problems common to all 
cancers, such as cancer-related pain, poor quality of life and the long-term side effects of cancer treatment, patients with oral cancer may also suffer from facial deformity and oral dysfunction. These unique characteristics may cause and aggravate patients' mental distress, such as depression, anxiety and posttraumatic stress disorder (PTSD). ${ }^{6}$ However, while depression and anxiety are known to be prevalent in patients with cancer, PTSD symptoms have been examined less frequently in this population.

PTSD is a continued mental disturbance that affects individuals after they have witnessed, experienced or encountered a traumatic event with a risk of serious injury or death. ${ }^{7}$ PTSD has three typical symptoms: intrusion (mentally re-experiencing the trauma), avoidance (such as thoughts, persons, places or situations related to the traumatic event), and hypervigilance (always being alert, difficulty concentrating). Conceptually, cancer is different from other known PTSD stressors, such as war, traffic accidents, rape, and natural disasters. ${ }^{8}$ However, oral cancer treatment can be intrusive, resulting in permanent functional and disfiguring changes, thereby increasing the likelihood of a posttraumatic stress reaction. ${ }^{9}$ In addition, cancer has been identified as a potential trauma in the Diagnostic and Statistical Manual of Mental Disorders Fourth Edition (DSM-IV). ${ }^{10}$ PTSD symptoms are associated with impaired overall health, quality of life and survival in cancer patients. ${ }^{8}$ Thus, it is imperative to investigate the prevalence of PTSD symptoms and to identify potential influencing factors.

Stress, defined as a complex physiological state that embodies a range of integrative physiological and behavioural processes which occur when there is a real or perceived threat to homeostasis, can affect many aspects of life (ie, physical, behavioural and psychiatric manifestations). ${ }^{11}$ The potential stresses of cancer patients are widely acknowledged. Patients diagnosed with cancer are exposed to a high level of stress that derives from lifethreatening feelings, an unpredictable survival time, and the crushing cost of health care. In addition, compared with other types of cancer, oral cancer patients are faced with multiple stressors, including significant changes in body image and altered oral function. Besser et al found that people who had experienced high levels of stress expressed high levels of PTSD symptoms, ${ }^{12}$ which means that perceived stress is associated with PTSD symptoms. Therefore, it is of great importance to study the impact of stress on the PTSD symptoms of patients with oral cancer.
As a positive psychological resource, hope has become of increasing importance in clinical practice. Hope is defined as a multidimensional dynamic life force characterized by a confident yet uncertain expectation of achieving a future good which is realistically possible and personally significant.

${ }^{13}$ Based on this definition, Herth developed a model of hope to measure the hope level in cancer patients, which consisted of three dimensions: temporality and future; positive readiness and expectancy; and interconnectedness. ${ }^{14}$ A previous study reported that hope played an important role in tapering off stress and PTSD symptoms. ${ }^{15}$ Liu et al also measured the relationship between hope and PTSD symptoms in patients who were diagnosed with haematological malignancies and found a negative correlation between hope and PTSD symptoms. $^{8}$

Moreover, in many studies, hope was often regarded as a mediator to combat PTSD symptoms. For example, Zhou et al found that hope mediated the relationship between social support and PTSD symptoms among adolescents following the Ya'an earthquake. ${ }^{16}$ A previous study also found that hope partially mediated the relationship between social support and PTSD symptoms among Chinese patients with ovarian cancer. ${ }^{17}$ Therefore, in the present study, we aimed to investigate the relationship between hope and PTSD symptoms and to examine the mediating effect of hope among Chinese patients with oral cancer.

In light of the above concerns, the aims of our study were: (1) to explore the prevalence of PTSD symptoms and its associated factors among Chinese patients with oral cancer, (2) to examine the relationship of perceived stress, hope and PTSD symptoms among Chinese patients with oral cancer, and (3) to determine the mediating role of hope on the association between perceived stress and PTSD symptoms.

\section{Patients and Methods}

\section{Study Design and Sampling}

A cross-sectional study was conducted in Shenyang, China, from May 2016 to October 2017. The participants were sampled through a convenience sampling method. They were recruited from two hospitals in Liaoning Province, including the Shengjing Hospital of China Medical University and the Stomatology Hospital of China Medical University. Once patients agreed to 
participate in this study, they were given the questionnaires and informed consent forms to read and sign. The eligibility criteria were as follows: the patients were over 18 years old, had been clinically diagnosed with oral cancer, had already undergone surgical resection of their cancer, were aware of their own diagnosis, were well enough to fill out the questionnaires and had the ability to accurately and fluently answer questions. The exclusion criteria included patients that had a history of psychiatric or cognitive disorders (such as depression or anxiety), were illiterate and unable to complete the survey, and had other oral diseases or other cancers. Eligible patients were invited to join the study by their attending physicians. The investigators were responsible for helping the patients understand all of the questionnaire items without any inducement in regard to their answer. Finally, the completed questionnaires were obtained from 230 individuals, including 135 men and 95 women.

The present study received ethical approval from the Committee on Human Experimentation of China Medical University, and all of the research procedures were conducted in conformity to the ethical standards.

\section{Demographical and Clinical Characteristics}

The demographic characteristics collected about the patients included age, gender, marital status, educational level, monthly income, work status, and residence. Their age was divided into two ranges: $<60$ and $\geq 60$ years. Their marital status was categorized as married/cohabitation, single/divorced/separated/widowed. Options for educational level included "middle school or lower", "high or secondary school" and "college or above". Monthly income was categorized as " $<3000 \mathrm{RMB}$ " and " $\geq 3000$ RMB". Work status was categorized as "retirement/unemployed", "temporary worker" and "regular employee". Residence was categorized as "rural area" and "urban area". Clinical characteristics of their cancer included familial inheritance and distant metastasis.

\section{Measurements}

The Chinese version of the Posttraumatic Stress Checklist-Civilian Version (PCL-C) was used to measure PTSD symptoms, including avoidance, intrusion and hyperarousal, according to the DSM-IV symptom criteria. ${ }^{18}$ The PCL-C consists of 17 items, and each item uses a 5-point Likert scale (from 1 "not at all" to
5 "extremely") to reflect the severity of a particular symptom in the past month. The total score ranges from 17 to 85, with a higher total score indicating more severe PTSD symptoms. A total score $\geq 50$ suggests the probable presence of PTSD. The Chinese version of the PCL-C has shown adequate reliability and validity in cancer patients. ${ }^{19,20}$ In the present study, the Cronbach's $\alpha$ for the total scale was 0.950 .

The Perceived Stress Scale-10 (PSS-10) was used to assess perceived stress. ${ }^{21}$ This scale was used to assess the level of perceived stress in people's lives. The item is scored on a 5-point Likert scale ranging from never to very often. The total score ranges from 0 to 40 , and higher scores indicate a higher level of perceived stress. The Chinese version has demonstrated good reliability and validity. ${ }^{22}$ Its Cronbach's $\alpha$ was 0.833 in this study.

The Herth Hope Index (HHI) was used to assess the level of hope in the patients. This scale has 12 items, and each item has 4 response categories from 1 to $4 .^{23}$ A higher total score reflects a higher level of hope. The Chinese version of the HHI has been widely used in cancer patients with good reliability and validity. ${ }^{24}$ In the present study, the Cronbach's $\alpha$ of the total scale was 0.809 .

\section{Statistical Analysis}

All statistical analyses were conducted using the Statistical Package for the Social Sciences (SPSS, version 23.0), with a two-tailed probability value of $<$ 0.05 considered to be statistically significant. The demographic and clinical variables are described as the mean, standard deviation (SD), number (n) and percentage (\%), as appropriate. The distribution of PTSD symptoms in the categorical, demographic and clinical variables was tested by $t$-tests or one-way ANOVA. The correlations among PTSD, perceived stress and hope were conducted using Pearson's correlation analysis. Hierarchical multiple linear regression analysis was performed to explore the mediation of hope in the relationship between perceived stress and PTSD symptoms. Control variables (educational level, smoking, drinking and distant metastasis) were entered in step 1. In step 2, perceived stress was included as an independent variable. Hope was added in step 3.

We use asymptotic and resampling strategies to examine hope as a potential mediating role in the association between perceived stress and PTSD symptoms. ${ }^{25}$ As shown in 
Step 1

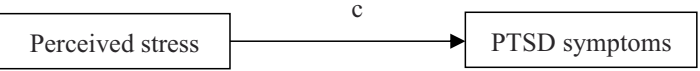

Step 2

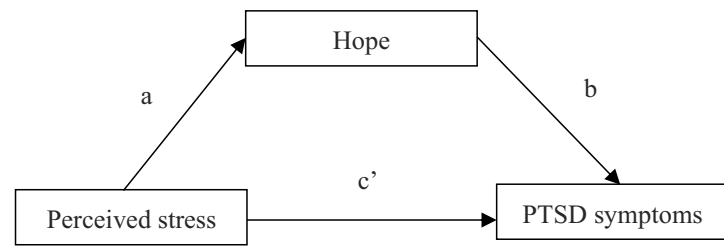

Figure I Theoretical model of the mediation of hope on the association between perceived stress and PTSD symptoms. c: total effect of perceived stress on PTSD symptoms; a: association of perceived stress with hope; b: association between hope and PTSD symptoms after controlling for the covariate; c': direct effect of perceived stress on PTSD symptoms after adding hope ad mediator.

Figure 1, the aim of step 1 was to test whether the relationship between perceived stress and PTSD symptoms was significant (path c) while the aim of step 2 was to examine the mediation of hope (path $\mathrm{a}^{*} \mathrm{~b}$ ). If the direct effect (path c') of perceived stress on PTSD symptoms in step 2 was smaller than the total effect (path c) in step 1 or was not significant, the mediation of hope possibly existed. In this study, we used 5000 bootstrap samples. A bias-corrected and accelerated 95\% confidence interval ( $\mathrm{BCa} 95 \% \mathrm{CI}$ ) was conducted for each $\mathrm{a} * \mathrm{~b}$ product, and a $\mathrm{BCa} 95 \% \mathrm{CI}$ excluding 0 indicated significant mediation. The PTSD, HHI, and PSS scores were standardized to account for the differences in the scale scores.

\section{Results}

\section{Descriptive Statistics}

In the present study, the demographic and clinical characteristics of the patients and the PTSD symptom scores for the different categories of variables are shown in Table 1. A total of 230 patients with oral cancer participated in our survey, including 135 men and 95 women, with a mean (SD) age of 56.13 (13.54) years old. More than half of the participants had a household monthly income under 3000 RMB. Approximately $89.13 \%$ of the patients were married or cohabiting. Additionally, only approximately $11.74 \%$ of the participants had an educational level of college or above. Concerning the clinical variables, 16 (6.95\%) of the patients had familial inheritance of cancer, and 13 $(5.65 \%)$ of the patients had distant metastasis.

Among all of the variables, the PCL-C scores were significantly different among the different categories of variables, including educational background $(F=4.507$, $p=0.012)$, smoking $(t=3.028, p=0.003)$, alcohol consumption $(t=2.534, p=0.012)$ and distant metastasis $(t=$
3.365, $p=0.001)$. However, the differences in the other variables were not statistically significant.

\section{Correlations Among PTSD Symptoms, Perceived Stress and Hope}

The correlation analyses of the continuous variables are presented in Table 2. The mean PCL-C score among patients with oral cancer was $32.20 \pm 11.04$. Perceived stress was significantly and positively associated with PTSD symptoms ( $r=0.645, p<0.001)$, whereas hope was significantly and negatively associated with PTSD symptoms $(r=-0.628, p<0.001)$. Perceived stress was negatively associated with hope $(r=-0.363, p<0.001)$.

\section{Hierarchical Multiple Regression Analysis}

All of the independent variables that were associated with PTSD symptoms in the univariate analysis $(p<0.05)$ were entered into the hierarchical multiple regression model. As shown in Table 3, in step 1, control variables including educational level, smoking, drinking alcohol and distant metastasis as a whole accounted for $5.6 \%$ of the variance in PTSD symptoms. In step 2, after controlling for the control variables, perceived stress was significantly and positively associated with PTSD symptoms $(\beta=0.659, \mathrm{p}<0.001)$. In step 3 , hope was negatively correlated with the PTSD symptoms $(\beta=$ $-0.309, \mathrm{p}<0.001$ ), and the effect of perceived stress on the PTSD symptoms was smaller $(\beta=0.477, \mathrm{p}<0.001)$, which indicated that hope probably plays a mediating role in the relationship between perceived stress and PTSD symptoms.

\section{The Mediating Role of Hope Between Perceived Stress and PTSD Symptoms}

As shown in Figure 2, the total effect (path c) of perceived stress on PTSD and the indirect effect of perceived stress on the PTSD symptoms via hope (path $\mathrm{a}^{*} \mathrm{~b}$ ) were evaluated. The direct effect (path c') of perceived stress on the PTSD symptoms was still significant when hope was included as a mediator, and the $\mathrm{BCa} 95 \%$ CI did not include 0 . Therefore, hope had a partially mediating effect on the relationship between perceived stress and PTSD symptoms $(\mathrm{a} * \mathrm{~b}=-0.46$; BCa 95\% CI: 0.285, 0.655). The proportion of the total effect of perceived stress on PTSD symptoms mediated by hope was calculated with the formula $\left(a^{*} b\right) / c$ to assess the mediating effect size. The proportion of hope was $30.06 \%$ in the present study. 
Table I Demographic and Clinical Characteristics of Participants and Result of Univariate Analysis

\begin{tabular}{|c|c|c|c|c|c|}
\hline \multirow{2}{*}{ Variables } & \multirow[t]{2}{*}{$\mathbf{N}(\%)$} & \multicolumn{4}{|l|}{ PTSD } \\
\hline & & Mean & SD & $\mathbf{F} / \mathbf{t}$ & P-value \\
\hline Gender & & & & 1.870 & 0.063 \\
\hline Male & $135(58.6)$ & 33.34 & 11.12 & & \\
\hline Female & $95(4 I .4)$ & 30.58 & 10.78 & & \\
\hline Age & & & & 0.444 & 0.658 \\
\hline$<60$ & $139(60.4)$ & 32.55 & $|1.4|$ & & \\
\hline$\geq 60$ & $91(39.6)$ & 31.90 & 10.75 & & \\
\hline Marital status & & & & 1.775 & 0.077 \\
\hline Single/divorced/ & $25(10.8)$ & 28.52 & 10.51 & & \\
\hline Separated/widowed & & & & & \\
\hline Married/cohabitation & 205(89.2) & 32.65 & 11.04 & & \\
\hline Residence & & & & 0.965 & 0.336 \\
\hline Rural area & $145(63.1)$ & 31.67 & 10.65 & & \\
\hline Urban area & $85(36.9)$ & 33.13 & 11.69 & & \\
\hline Educational background & & & & 4.507 & 0.012 \\
\hline Middle school or lower & $101(43.9)$ & 30.92 & 9.42 & & \\
\hline High or secondary school & $102(44.3)$ & 34.42 & 12.05 & & \\
\hline College or above & $27(11.7)$ & 28.26 & 11.45 & & \\
\hline Household monthly income (yuan) & & & & 1.094 & 0.275 \\
\hline$<3000$ & $145(63.0)$ & 32.80 & 10.96 & & \\
\hline$\geq 3000$ & $85(37)$ & 31.14 & 11.17 & & \\
\hline Job status & & & & 0.410 & 0.664 \\
\hline Retirement/unemployed & $133(57.8)$ & 32.23 & 10.78 & & \\
\hline Temporary workers & $32(13.9)$ & 33.64 & 10.35 & & \\
\hline Regular employee & $65(28.2)$ & 31.45 & 11.98 & & \\
\hline Smoking & & & & 3.028 & 0.003 \\
\hline Yes & $118(51.3)$ & 30.09 & 10.93 & & \\
\hline No & $112(48.7)$ & 34.42 & 10.76 & & \\
\hline Drinking alcohol & & & & 2.534 & 0.012 \\
\hline Yes & $135(58.7)$ & 30.67 & 10.75 & & \\
\hline No & $95(4 I .3)$ & 34.37 & 11.14 & & \\
\hline Familial inheritance & & & & 1.273 & 0.204 \\
\hline Yes & $16(6.9)$ & 32.34 & 11.13 & & \\
\hline No & $214(93.4)$ & 28.60 & 8.90 & & \\
\hline Distant metastasis & & & & 3.365 & 0.001 \\
\hline Yes & $13(5.6)$ & 42.00 & 14.11 & & \\
\hline No & $217(94.4)$ & 31.62 & 10.59 & & \\
\hline
\end{tabular}

Abbreviation: SD, standard deviation.

\section{Discussion}

This cross-sectional study of patients with oral cancer was conducted in Liaoning Province in the northeastern region of China, and it is the first in China to examine the association between perceived stress and PTSD symptoms and the mediating role of hope in this relationship. In this study on
PTSD symptoms in patients with oral cancer, based upon the scoring method (PCL-C scored 50 and above), $6.05 \%$ of our sample reported PTSD symptoms. This prevalence was similar to that of patients with gynaecologic cancer $(6.08 \%)$ in China ${ }^{26}$ but lower than the previously reported prevalence $(10.3 \%)$ of oral cancer in China. ${ }^{27}$ This difference 
Table 2 Correlations Among PTSD, Hope and Perceived Stress

\begin{tabular}{|l|l|l|l|l|l|}
\hline & Mean & SD & PTSD & Hope & Perceived Stress \\
\hline PTSD & 32.20 & 11.04 & 1 & & \\
Hope & 36.42 & 4.63 & $-0.628^{* *}$ & 1 & \\
Perceived stress & 16.67 & 4.83 & $0.645^{* *}$ & $-0.363^{* *}$ & 1 \\
\hline
\end{tabular}

Note: **p $<0.01$.

Abbreviation: SD, standard deviation.

Table 3 Hierarchical Multiple Regression Analysis Results

\begin{tabular}{|c|c|c|c|c|c|c|}
\hline \multirow[t]{2}{*}{ Variables } & \multicolumn{2}{|l|}{ Step I } & \multicolumn{2}{|l|}{ Step 2} & \multicolumn{2}{|l|}{ Step 3} \\
\hline & $\beta$ & $P$-value & $\beta$ & $P$-value & $\beta$ & $P$-value \\
\hline Control variables & & & & & & \\
\hline Edu-I & -0.176 & 0.010 & -0.063 & 0.219 & -0.054 & 0.260 \\
\hline Edu-2 & -0.124 & 0.065 & -0.022 & 0.673 & 0.061 & 0.951 \\
\hline Smoking & 0.097 & 0.245 & 0.103 & 0.100 & 0.084 & 0.153 \\
\hline Drinking alcohol & 0.090 & 0.275 & 0.173 & 0.006 & 0.149 & 0.011 \\
\hline Distant metastasis & 0.201 & 0.002 & 0.136 & 0.005 & 0.080 & 0.087 \\
\hline Perceived stress & & & 0.659 & $<0.000$ & 0.477 & $<0.000$ \\
\hline Hope & & & & & -0.309 & $<0.000$ \\
\hline$F$ & $5.632^{* *}$ & & $38.025 * * *$ & & $41.464 * * *$ & \\
\hline$R^{2}$ & 0.112 & & 0.506 & & 0.567 & \\
\hline Adjusted $R^{2}$ & 0.092 & & 0.492 & & 0.553 & \\
\hline$R^{2}$-change & 0.112 & & 0.394 & & 0.061 & \\
\hline
\end{tabular}

Notes: Edu-I means “middle school or lower" vs "college or above"; Edu-2 means "high or secondary school" vs "college or above"; **p <0.0I, ***p <0.00I.

may be attributed to the difference in measures and diagnostic criteria. Using the same diagnostic tool (PCL-C) and the same cut-off score $(\geq 50)$, the PTSD symptom prevalence was determined to be between $1.9 \%$ and $12 \%$ among
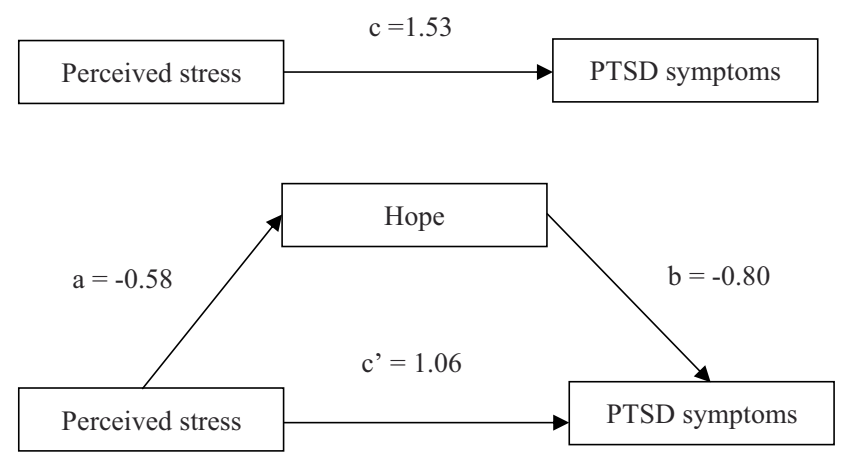

Figure 2 Model of the mediation of hope on the association between perceived stress and PTSD. c: total effect of perceived stress on PTSD symptoms $(c=1.53$, $p<0.0 \mathrm{I})$; a: association of perceived stress with hope $(a=-0.58, p<0.0 \mathrm{I})$; b: association between hope and PTSD symptoms after controlling for the covariate $(b=-0.80, p<0.01)$; $c^{\prime}$ : direct effect of perceived stress on PTSD symptoms after adding hope ad mediator $\left(c^{\prime}=1.06, p<0.01\right)$. Educational level, smoking, drinking alcohol, familial inheritance and distant metastasis were covariates. patients with breast cancers. ${ }^{28-31}$ Among head and neck cancer survivors, 11 of 93 participants $(11.8 \%)$ who scored $\geq 44$ on the PCL-C demonstrated PTSD. ${ }^{32}$ Moreover, studies using self-reported PTSD symptom measures yielded a prevalence ranging from $7.3 \%$ to $13.8 \%$, and using more stringent, clinician-administered structured diagnostic interviews for PTSD yielded a current prevalence estimated at $6.4 \%$ (95\% CI 4.1-9.9). ${ }^{33,34}$ In fact, because the knowledge about PTSD symptoms in oral cancer is relatively limited and there are differences in the measurement tools and diagnostic methods, a clear and reasonable comparison of the prevalence of PTSD could not be carried out. However, the present study clearly demonstrated that some patients with oral cancer suffered from clinically significant symptoms of posttraumatic stress. Therefore, it is imperative to determine the crucial influencing factors and identify targeted solutions.

Regarding demographic and clinical factors, patients with a college degree or above had the lowest PCL-C score. In contrast, patients with distant metastasis had the highest 
PCL-C scores. This is probably due to the fact that distant metastasis means that patients suffer from persistent intrusive re-experiences of harrowing treatment procedures, which is a well-known risk factor for cancer-related PTSD. ${ }^{35}$ Moreover, patients with greater educational advantages might more clearly understand their illness, which could reduce illness uncertainty ${ }^{36}$ since illness uncertainty has been proven to be positively associated with PTSD symptoms. $^{37}$

The results of this study showed that after controlling for the covariates, perceived stress and hope were the key factors affecting PTSD symptoms, explaining $45.5 \%$ of the total variance. Specifically, perceived stress was found to be positively related to PTSD symptoms, which echoes the findings of similar studies. ${ }^{38-40}$ The potential stress of cancer patients is widely acknowledged since a cancer diagnosis is a stressful event for most individuals. ${ }^{1}$ In addition to the overload of physiological stress caused by the cancer and its treatment, many patients experience mental stress, such as worries about their prognosis and treatments, disruption of their ordinary life functions and an uncertain survival time. On the other hand, compared with other types of cancer, patients with oral cancer always face substantial challenges, including problems with speaking, eating, breathing, and facial disfigurement. Functional and facial changes have been proven to have an adverse impact on patients' psychological well-being and to aggravate their stress level. ${ }^{2}$ Thus, it is urgent to enact effective strategies to reduce cancer-related stress levels in patients with oral cancer.

With regard to hope, we found that it was negatively correlated with PTSD symptoms in patients with oral cancer, which was similar to the findings of prior studies. $^{6,41,42}$ A meta-analytic review conducted by Gallagher found that hope had a more strongly negative relationship with PTSD symptoms than with other positive expectancies. ${ }^{43}$ This might be mainly caused by the theory of hope interacting with PTSD symptoms. Patients who suffer from PTSD often feel if they do not have the ability to cope with stressors in their lives and expect negative outcomes when exposed to feared situations. However, hope could provide individuals with a positive resource to combat those psychological health-related problems and build confidence in their abilities. Meanwhile, hope has also been found to help patients adapt to cancer and give cancer patients directions and reasons for survival, ${ }^{44}$ which could help patients reduce their PTSD symptoms.
Thus, one way to alleviate PTSD symptoms is to improve the level of hope among Chinese patients with oral cancer.

As expected, hope was found to play partial mediating roles in the relationship between perceived stress and PTSD symptoms. Our results suggested that perceived stress could reduce the level of hope of Chinese patients with oral cancer; subsequently, reduced hope could lead to their PTSD symptoms. In other words, we could reduce or prevent PTSD symptoms in patients with oral cancer by enhancing the level of hope and/or decreasing the level of perceived stress. This result is in accordance with previous studies that verified the mediating effect of hope in relationships among many cancer patients' psychological outcomes. $^{44-46}$ Compared to decreasing patients' cancerrelated stress, it is more feasible for healthcare providers to develop interventions that increase the hope level of oral cancer patients. According to Luthans' psychological capital theory, hope is a positive psychological resource that can be readily measured, developed and effectively managed. Interventions designed to enhance the level of hope have been introduced in previous studies and confirmed to be effective. ${ }^{42,47}$ Although positive resources are receiving more attention in the oncology field, there are still few psychological interventions involving hope therapy. Therefore, future studies should focus more on this area. Thus, hope therapy should be included in interventions for oral cancer patients with PTSD symptoms.

Several limitations in this study design have to be taken into account when the results are evaluated. First, because of the cross-sectional design and self-reported measures, no conclusion could be drawn about a causal relationship between perceived stress and PTSD symptoms. Second, this study was conducted in one city from one province of China. The representativeness of the sample might thus be affected. Third, several potential clinical factors, such as recurrence, pathological stage and mode of treatment received, may affect the PTSD symptoms of patients with oral cancer, but we did not include them in our study. Finally, this study focused only on the associations among hope, perceived stress and PTSD symptoms, other factors such as social support, resilience and selfefficacy needed further consideration. Thus, a more comprehensive study should be conducted in the future.

\section{Conclusion}

In conclusion, our study showed that $6.05 \%$ of Chinese patients with oral cancer had PTSD symptoms. Perceived stress was positively associated with PTSD symptoms, and 
hope was negatively associated with PTSD symptoms. Furthermore, hope partially mediated the relationship between perceived stress and PTSD symptoms. Thus, more attention should be paid to patients' psychological status. Interventions concerning perceived stress and hope should be implemented to prevent or reduce PTSD symptoms among Chinese patients with oral cancer.

\section{Abbreviations}

PTSD, post-traumatic stress disorder; PCL-C, Posttraumatic Stress Checklist-Civilian Version; PSS-10, The Perceived Stress Scale-10; HHI, Herth Hope Index; ANOVA, analysis of variances; SCC, squamous cell carcinomas; SD, standard deviation.

\section{Data Sharing Statement}

The datasets analyzed during the current study are available from the corresponding author on reasonable request.

\section{Ethics Approval and Informed Consent}

All patients provided their informed written consent. This study was approved by the Committee on Human Experimentation of China Medical University. And this study complied with the Declaration of Helsinki.

\section{Consent for Publication}

No applicable.

\section{Acknowledgments}

We would like to thank all the patients who voluntarily participated in this study and research assistants who performed the data collection.

\section{Author Contributions}

All authors made a significant contribution to the work reported, whether that is in the conception, study design, execution, acquisition of data, analysis and interpretation, or in all these areas; took part in drafting, revising or critically reviewing the article; gave final approval of the version to be published; have agreed on the journal to which the article has been submitted; and agree to be accountable for all aspects of the work.

\section{Funding}

There were no funding sources.

\section{Disclosure}

There were no competing interests.

\section{References}

1. Gao Y, Yuan L, Pan B, Wang L. Resilience and associated factors among Chinese patients diagnosed with oral cancer. BMC Cancer. 2019;19(1):1-9. doi:10.1186/s12885-019-5679-0

2. Montero PH, Patel SG. Cancer of the oral cavity. Surg Oncol Clin N Am. 2015;24(3):491-508. doi:10.1016/j.soc.2015.03.006

3. Bray F, Ferlay J, Soerjomataram I, Siegel RL, Torre LA, Jemal A. Global cancer statistics 2018: GLOBOCAN estimates of incidence and mortality worldwide for 36 cancers in 185 countries. $C A-A$ CANCER J Clin. 2018;68(6):394-424. doi:10.3322/caac.21492

4. Chen W, Sun K, Zheng R, et al. Cancer incidence and mortality in China, 2014. Chinese J Cancer Res. 2018;30(1):1-12. doi:10.21147/j. issn.1000-9604.2018.01.01

5. Wong TSC, Wiesenfeld D. Oral cancer. Aust Dent J. 2018;63:S91S99. doi:10.1111/adj.12594

6. Yang YL, Liu L, Li MY, Shi M, Wang L. Psychological disorders and psychosocial resources of patients with newly diagnosed bladder and kidney cancer: a cross-sectional study. PLoS One. 2016;11(5):1-17. doi:10.1371/journal.pone. 0155607

7. Andreasen NC. What is post-traumatic stress disorder? Dialogues Clin Neurosci. 2011;13(3):240-243.

8. Liu L, Yang YL, Wang ZY, Wu H, Wang Y, Wang L. Prevalence and positive correlates of posttraumatic stress disorder symptoms among Chinese patients with hematological malignancies: a cross-sectional study. PLoS One. 2015;10(12):1-15. doi:10.1371/journal. pone. 0145103

9. Chan CMH, Ng CG, Taib NA, Wee LH, Krupat E, Meyer F. Course and predictors of post-traumatic stress disorder in a cohort of psychologically distressed patients with cancer: A 4-year follow-up study. Cancer. 2018;124(2):406-416. doi:10.1002/cncr.30980

10. Mendelson G. Diagnostic and Statistical Manual of Mental Disorders, fourth edition (DSM-IV). Aust $N \quad Z \quad J$ Psychiatry. 1995;29 (3):529-535. doi:10.3109/00048679509064964

11. Tilbrook AJ, Neuropeptides, stress-related. In: Fink G, editor. Encyclopedia of Stress (Second Edition Second ed. Academic Press;2007:903-908. doi:10.1016/B978-012373947-6.00735-2

12. Besser A, Neria Y, Haynes M. Adult attachment, perceived stress, and PTSD among civilians exposed to ongoing terrorist attacks in Southern Israel. Pers Individ Dif. 2009;47(8):851-857. doi:10.1016/j. paid.2009.07.003

13. Dufault K, Martocchio BC. Symposium on compassionate care and the dying experience. Hope: its spheres and dimensions. Nurs Clin North Am. 1985;20(2):379-391.

14. Herth K. Abbreviated instrument to measure hope: development and psychometric evaluation. $J$ Adv Nurs. 1992;17:1251-1259. doi:10.1111/j.1365-2648.1992.tb01843.x

15. Hirsch JK, Sirois FM. Hope and fatigue in chronic illness: the role of perceived stress. J Health Psychol. 2016;21(4):451-456. doi:10.1177/ 1359105314527142

16. Zhou X, Wu X, Zhen R. Self-esteem and hope mediate the relations between social support and post-traumatic stress disorder and growth in adolescents following the Ya'an earthquake. Anxiety, Stress Coping. 2018;31(1):32-45. doi:10.1080/10615806.2017.1374376

17. Liu CL, Liu L, Zhang Y, Dai XZ, Wu H. Prevalence and its associated psychological variables of symptoms of depression and anxiety among ovarian cancer patients in China: a cross-sectional study. Health Qual Life Outcomes. 2017;15. doi:10.1186/s12955-0170738-1.

18. Weathers F, Litz B, Herman D, Huska JA, Keane T. The PTSD Checklist (PCL): reliability, validity, and diagnostic utility. Pap Present Annu Conv Int Soc Trauma Stress Stud. 1993. 
19. Xia ZY, Yue K, Ting-ting Y, Su-hua S, Yu-hong H. The impact of acceptance of disability and psychological resilience on post-trau_matic stress disorders in patients with burns. 2014.

20. Song Y $\bar{H}$, Sun WY, Xu YS. The effect of psychological care for patients with post-traumatic stress disorder. 2015;4-7.

21. Cohen S. Perceived stress in a probability sample of the United States. In: The Social Psychology of Health. The Claremont Symposium on Applied Social Psychology. Sage Publications, Inc; 1988:31-67.

22. Zheng T, Qu W, Ge Y, Sun X, Zhang K. The joint effect of personality traits and perceived stress on pedestrian behavior in a Chinese sample. PLoS One. 2017;12(11):1-18. doi:10.1371/journal. pone. 0188153

23. Herth K. Development and refinement of an instrument to measure hope. Sch Inq Nurs Pract. 1991;5(1):39-51; discussion 53-6.

24. Li MY, Yang YL, Liu L, Wang L. Effects of social support, hope and resilience on quality of life among Chinese bladder cancer patients: a cross-sectional study. Health Qual Life Outcomes. 2016;14(1):1-9. doi:10.1186/s12955-016-0481-z

25. Preacher KJ, Hayes AF. Asymptotic and resampling strategies for assessing and comparing indirect effects in multiple mediator models. Behav Res Methods. 2008;40:879-891. doi:10.3758/BRM.40.3.879

26. Chen L, Li P, Tang T, Min L. Correlation between mental health and coping style in gynecological cancer patients. 2012:13-15. DOI:10.16016/j.1000-5404.2012.11.011

27. Li M, Zhang H, Lou Y. Analysis of posttraumatic stress disorder and its influencing factors in patients with oral cancer after reconstruction. 2019

28. Mehnert A, Koch U. Prevalence of acute and post-traumatic stress disorder and comorbid mental disorders in breast cancer patients during primary cancer care: a prospective study. Psychooncology. 2007;16(3):181-188. doi:10.1002/pon.1057

29. Cordova MJ, Mitch ÆJGÆ, Carol GÆ, Chang V, Spiegel ÆD. Breast cancer as trauma: posttraumatic stress and posttraumatic growth Published Online. 2007;308-319. doi:10.1007/s10880-007-9083-6

30. Andrykowski MA, Cordova MJ, McGrath PC, Sloan DA, Kenady DE. Stability and change in posttraumatic stress disorder symptoms following breast cancer treatment: a 1-year follow-up. Psychooncology. 2000;9(1):69-78. doi:10.1002/(SICI)1099-1611 (200001/02)9:1<69::aid-pon439>3.0.CO;2-R

31. Cordova M, Studts J, Hann D, Jacobsen P, Andrykowski M. Symptom structure of PTSD following breast cancer. J Trauma Stress. 2000;13:301-319. doi:10.1023/A:1007762812848

32. Moschopoulou E, Hutchison I, Bhui K, Korszun A. Post-traumatic stress in head and neck cancer survivors and their partners. Support Care Cancer. 2018;26(9):3003-3011. doi:10.1007/s00520-018-41469

33. Cordova MJ, Riba MB, Spiegel D. Post-traumatic stress disorder and cancer. The Lancet Psychiatry. 2017;4(4):330-338. doi:10.1016/ S2215-0366(17)30014-7

34. Abbey G, Thompson SBN, Hickish T, Heathcote D. A meta-analysis of prevalence rates and moderating factors for cancer-related post-traumatic stress disorder. Psychooncology. 2015;24 (4):371-381. doi:10.1002/pon.3654

Cancer Management and Research

Publish your work in this journal

Cancer Management and Research is an international, peer-reviewed open access journal focusing on cancer research and the optimal use of preventative and integrated treatment interventions to achieve improved outcomes, enhanced survival and quality of life for the cancer patient.
35. Wachen JS, Patidar SM, Mulligan EA, Naik AD, Moye J. Cancerrelated PTSD symptoms in a veteran sample: association with age, combat PTSD, and quality of life. 2015;23(8):921-927. doi:10.1002/ pon.3494.Cancer-related

36. Tackett AP, Cushing CC, Suorsa KI, et al. Illness uncertainty, global psychological distress, and posttraumatic stress in pediatric cancer: a preliminary examination using a path analysis approach. $J$ Pediatr Psychol. 2016;41(3):309-318. doi:10.1093/jpepsy/jsv093

37. Moreland P, Santacroce SJ, Illness uncertainty and posttraumatic stress in young adults with congenital heart disease. J Cardiovasc Nurs. 2018;33(4):356-362. doi:10.1097/JCN.0000000000000471

38. Lima BB, Hammadah M, Pearce BD, et al. Association of posttraumatic stress disorder with mental stress-induced myocardial ischemia in adults after myocardial infarction. JAMA Netw Open. 2020;3(4): e202734. doi:10.1001/jamanetworkopen.2020.2734

39. Asnakew S, Shumet S, Ginbare W, Legas G, Haile K. Prevalence of post-traumatic stress disorder and associated factors among Koshe landslide survivors, Addis Ababa, Ethiopia: a community-based, cross-sectional study. BMJ Open. 2019;9(6):1-8. doi:10.1136/bmjopen-2018-028550

40. Wang Q, Xu W, Ren L, Wang W, Wang Y. The relationship between hope and post-traumatic stress disorder in Chinese shidu parents: the mediating role of perceived stress. J Affect Disord. 2019;251 (September 2018):23-30. doi:10.1016/j.jad.2019.03.049

41. Nipp RD, El-jawahri A, Chan A, et al. HHS public access. 2019;124 (16):3445-3453. DOI:10.1002/cncr.31576.Symptoms

42. Damreihani N, Behzadipour S, Haghpanh S, Bordbar M. The effectiveness of positive psychology intervention on the well-being, meaning, and life satisfaction of mothers of children with cancer: a brief report. J Psychosoc Oncol. 2018;36(3):382-388. doi:10.1080/ 07347332.2018.1427173

43. Gallagher MW, Long LJ, Phillips CA. Hope, optimism, self-efficacy, and posttraumatic stress disorder: a meta-analytic review of the protective effects of positive expectancies. J Clin Psychol. 2020;76 (3):329-355. doi:10.1002/jclp.22882

44. Wang WL, Zhou YQ, Chai NN, Li GH, Liu DW. Mediation and moderation analyses: exploring the complex pathways between hope and quality of life among patients with schizophrenia. $B M C$ Psychiatry. 2020;20(1):1-9. doi:10.1186/s12888-020-2436-5

45. Tong C, Cui C, Li Y, Wang L. The effect of workplace violence on depressive symptoms and the mediating role of psychological capital in Chinese township general practitioners and nurses: a cross-sectional study. Psychiatry Investig. 2019;16(12):896-903. doi:10.30773/pi.2019.0095

46. Yao J, Yang L. Perceived prejudice and the mental health of Chinese ethnic minority college students: the chain mediating effect of ethnic identity and hope. Front Psychol. 2017;8(JUL):1-10. doi:10.3389/ fpsyg.2017.01167

47. Poorgholami F, Abdollahifard S, Zamani M, Kargar Jahromi M, Badiyepeymaiejahromi $\mathrm{Z}$. The effect of stress management training on hope in hemodialysis patients. Glob J Health Sci. 2015;8:165. doi:10.5539/gjhs.v8n7p165
The manuscript management system is completely online and includes a very quick and fair peer-review system, which is all easy to use. Visit http://www.dovepress.com/testimonials.php to read real quotes Visit http://www.dovepros published authors.
from

\section{Dovepress}

